\title{
Structural brain changes and depressive symptoms in older adults: Findings from 4 cohort studies
}

Ellen V Backhouse, Susan D Shenkin, Andrew Mclntosh, lan J Deary, Mark Bastin, Susanne de Rooij, Alison Murray, Tessa Roseboom \& Joanna M Wardlaw

Centre for Clinical Brain Sciences and Centre for Cognitive Ageing and Cognitive Epidemiology, University of Edinburgh, Edinburgh Dementia Research Centre in the UK Dementia Research Institute and the Academic Medical Centre, University of Amsterdam

\section{Background}

- Depressive symptoms are common in older people and are a major cause of morbidity and mortality.

- In older adults depressive symptoms are associated with brain atrophy and cerebral small vessel disease (cSVD), particularly white matter hyperintensities $(\mathrm{WMH})^{1}$.

- The impact of total cSVD burden on symptoms of depression has not been studied.

\section{Methods}

- Cohorts: community dwelling participants from STRADL $(n=280)$, the Dutch Famine Birth cohort $(n=118)$, the Lothian Birth Cohort $1936(n=685)$ and the Simpson cohort $(n=110)$.

- Brain Imaging: presence of each cSVD feature (analysed individually and summed to form a total "cSVD score", range 14), cortical infarcts, WMH volume and brain volumes (LBC 1936 and Simpson cohort only).

- Depressive symptoms: the Quick Inventory for Depressive Symptomatology (QIDS-16) (STRADL) and the Hospital Anxiety and Depression scale (HADS-D) (Dutch Famine Birth cohort, LBC 1936 and Simpson cohort).

- Statistics:, data analysed separately for each cohort using linear regression with bootstrapping and then meta-analysed, adjusted for age, sex, hypertension, smoking behaviour, adult socioeconomic status and cognition.

\section{Results}

The age of the cohorts was 62.3 (STRADL), 67.5 (Dutch Famine Birth cohort), 72.7 (LBC 1936), 78.4 (Simpson cohort).

Severity of total cSVD increased with increasing age of the cohorts but across all cohorts few had high CSVD scores (Fig. 1).

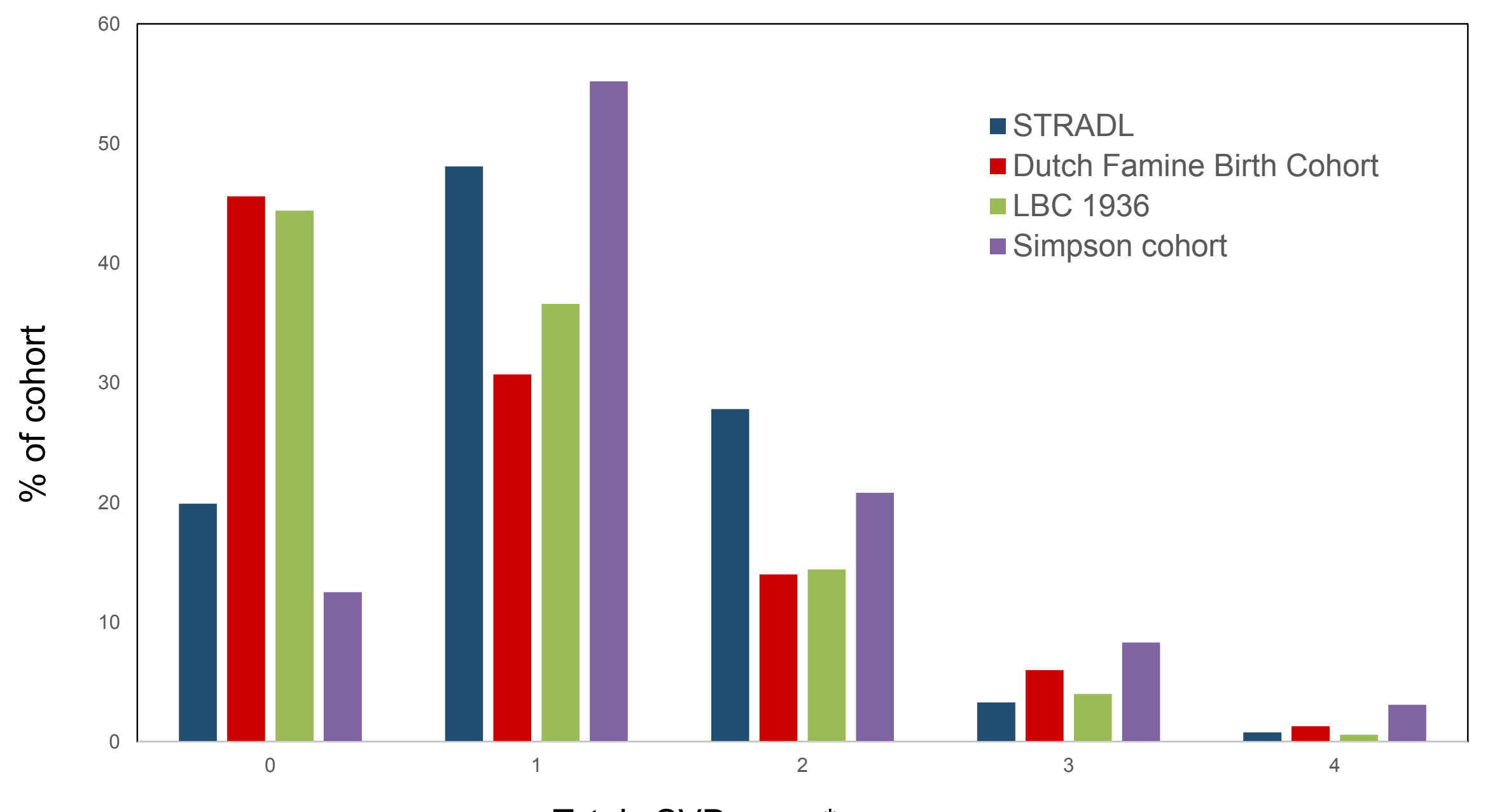

Figure 1: Breakdown of total CSVD score for STRADL, the Dutch Famine Birth Cohort, LBC 1936 and the Simpson cohort. ${ }^{* 1}$ point each for moderate/severe white matter hyperintensities, $1+$ lacune, $1+$ cerebral

Depressive symptoms on the HADS-D were higher in the Simpson cohort compared to the Dutch Famine Birth cohort and the LBC 1936 (Fig. 2).

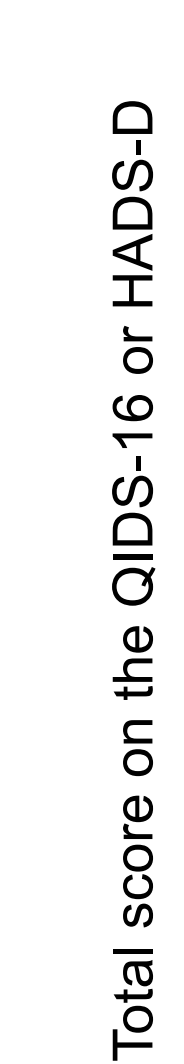

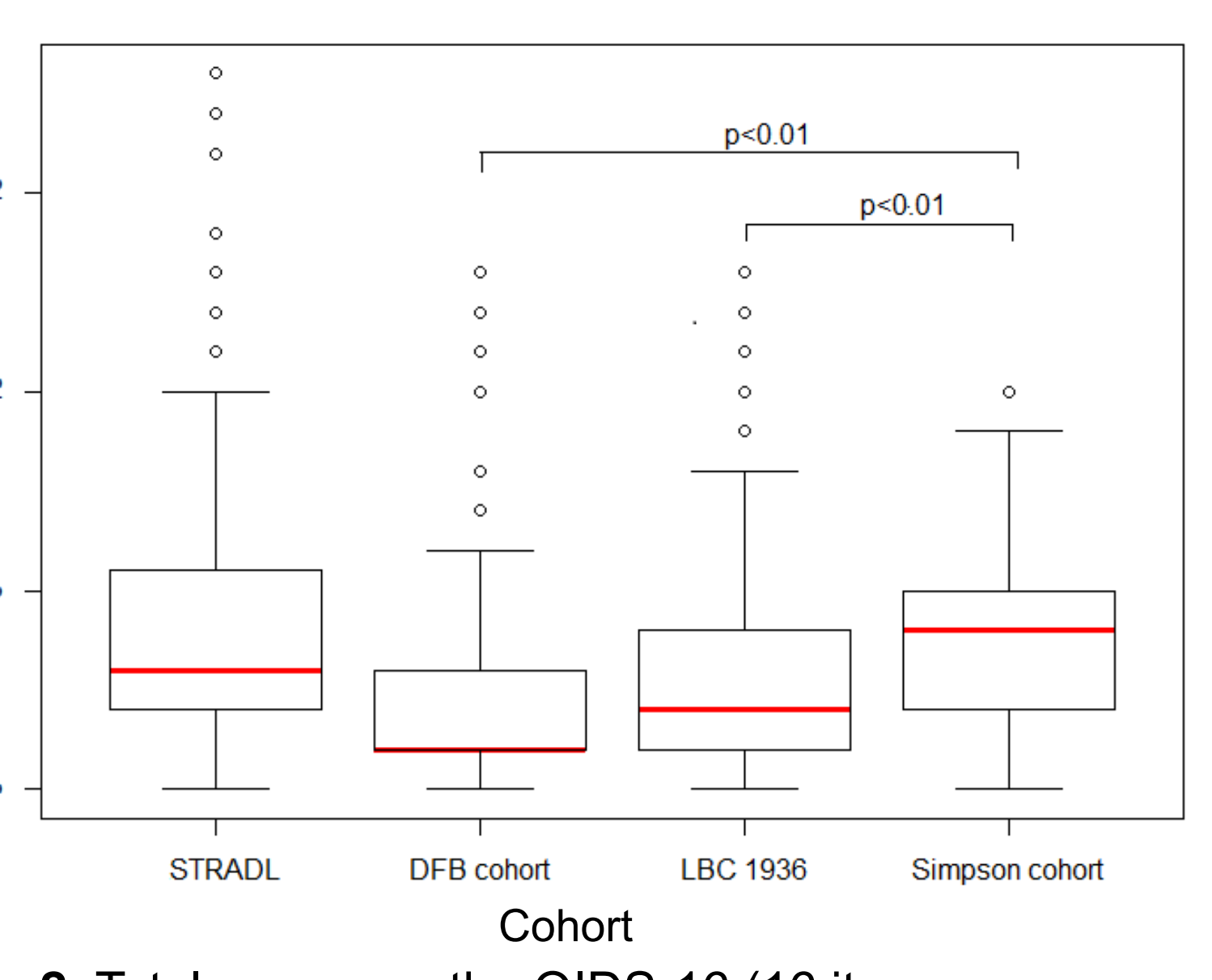

Figure 2: Total scores on the QIDS-16 (16 items score range 0-27) (STRADL) and the HADS-D (7 items range 0-21) (Dutch Famine Birth cohort the LBC 1936 and the Simpson cohor).

\section{All cohorts}

- Increased atrophy $(B=0.09, p=0.01$, Fig. 3B) and cortical infarcts $(B=0.08$, $p=0.02$, Fig. 3C) were associated with increased depressive symptoms.

\section{The LBC 1936 and Simpson cohort}

- $\mathrm{WMH}$ volume (Simpson cohort, $\mathrm{B}=0.24, \mathrm{p}=0.01$, Fig. 4A), atrophy (both cohorts, $B=-0.18, p=<0.001$, Fig. 4B) and whole brain volume (both cohorts, $B=-0.17, p=0.001$ ) were associated with decreased depressive symptoms (Fig. $4 C)$.

$(3 \mathrm{~A})$

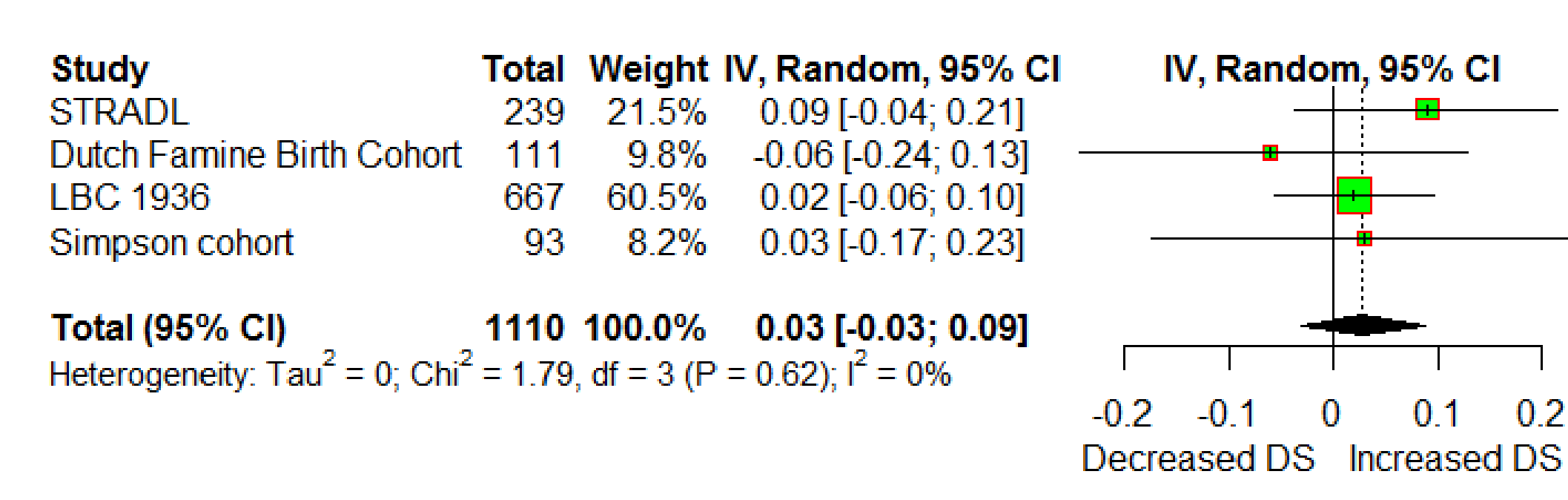

(3B)

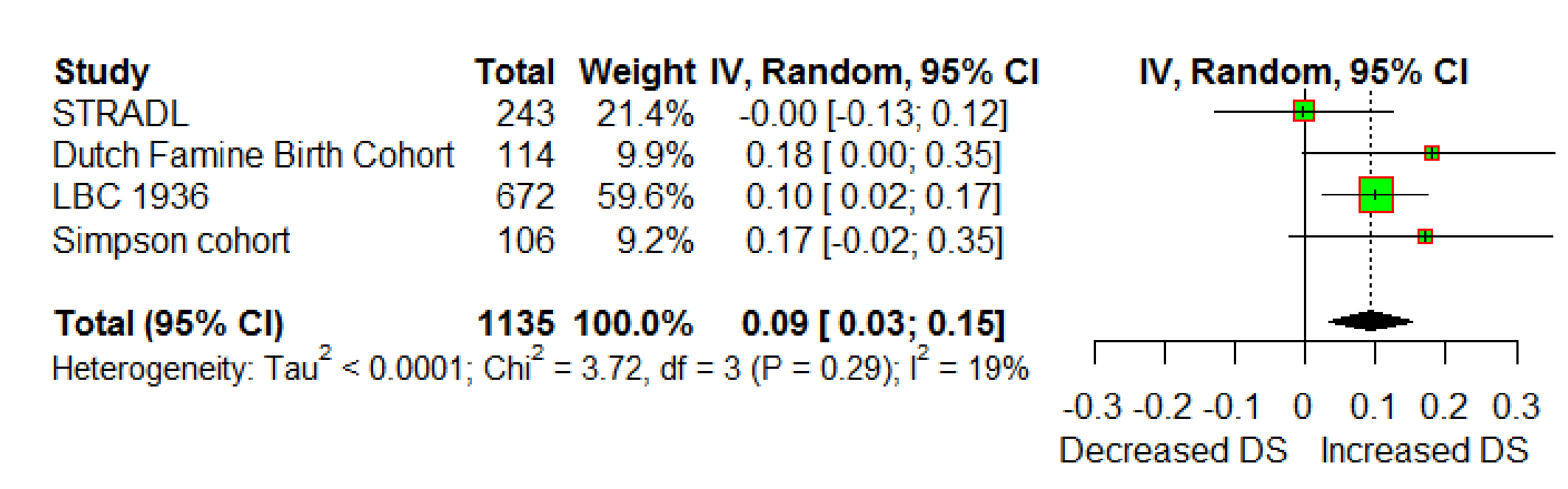

$(3 \mathrm{C})$

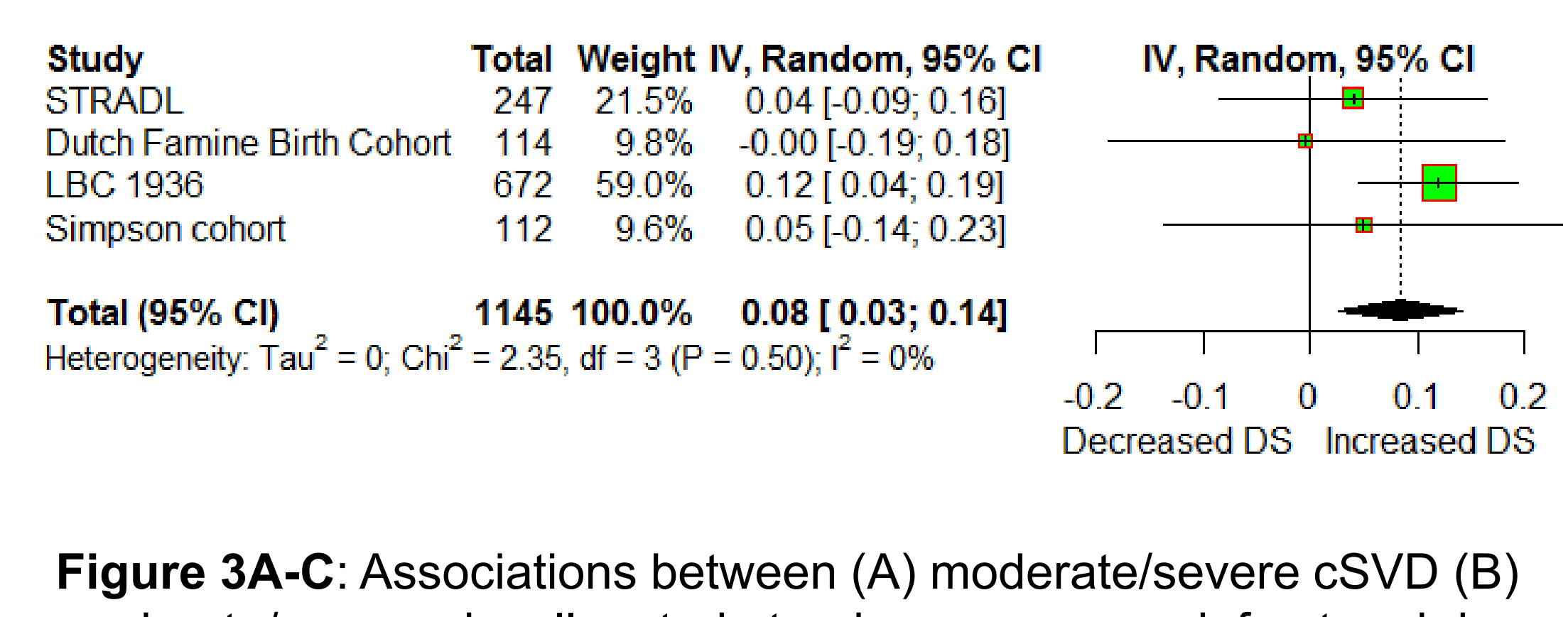

Figure 3A-C: Associations between (A) moderate/severe CSVD (B) moderate/se
symptoms
$(4 \mathrm{~A})$

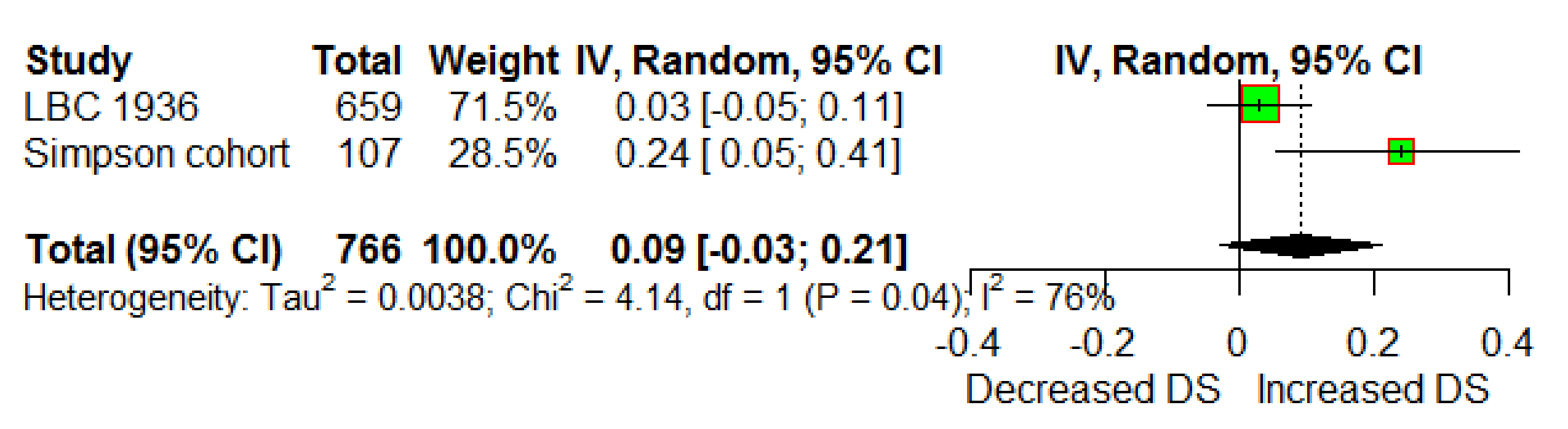

$(4 \mathrm{~B})$

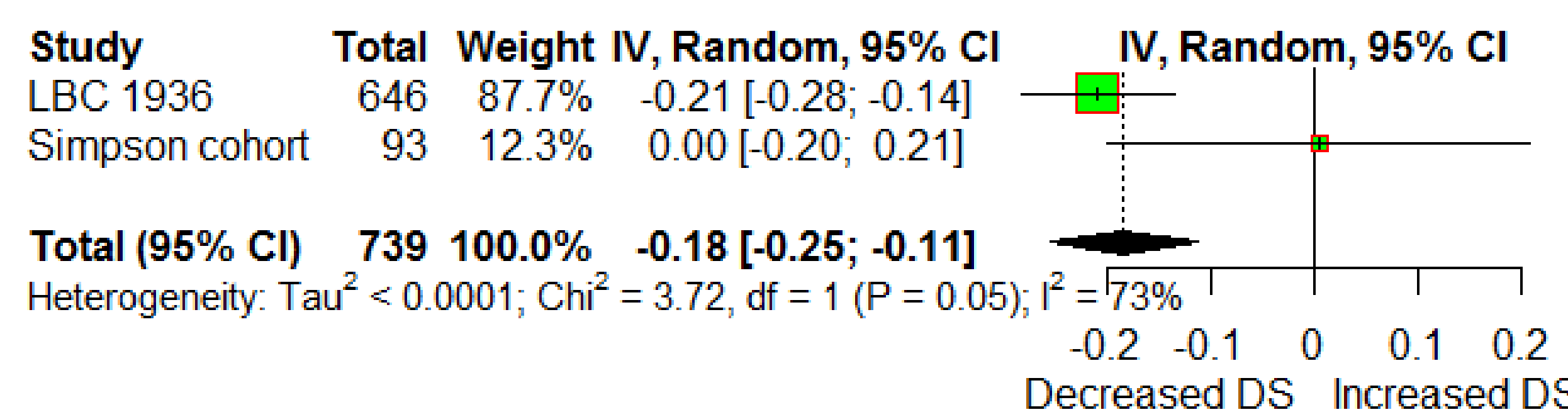

$(4 \mathrm{C})$

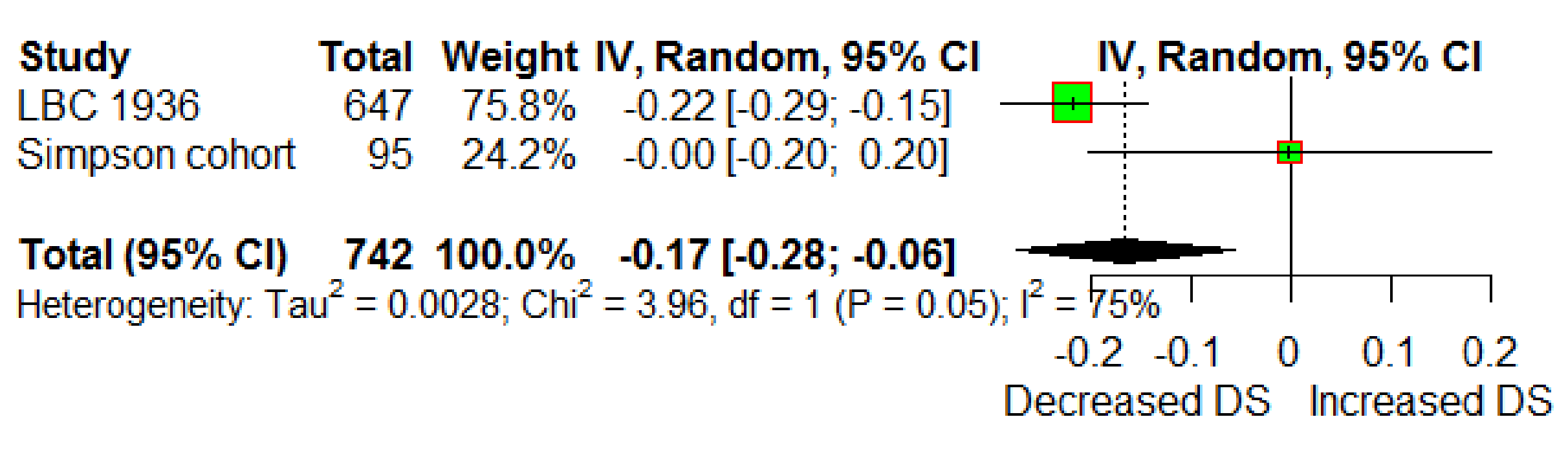

Figure 4A-C: Associations between (A) WMH volume (B) whole brain volume as a \% of the ICV (C) whole brain volume corrected for ICV and depressive symptoms.

\section{Conclusions}

These findings support an association between WMH, brain atrophy and depressive symptoms in older adults. It is unclear whether total cSVD burden is associated with depressive symptoms in these relatively healthy cohorts. Future studies should consider all features of CSVD, not just those presented here. Associations should be examined in those with more vascular disease and more depressive symptoms. 